\title{
Autophagy mediates the process of cellular senescence characterizing bile duct damages in primary biliary cirrhosis
}

\author{
Motoko Sasaki, Masami Miyakoshi, Yasunori Sato and Yasuni Nakanuma
}

Recent studies disclosed that autophagy is induced during and facilitates the process of senescence. Given that biliary epithelial cells (BECs) in damaged small bile ducts in primary biliary cirrhosis (PBC) show senescent features, we examined an involvement of autophagy in the process of biliary epithelial senescence in PBC. We examined immunohistochemically the expression of microtubule-associated proteins-light chain $3 \beta$ (LC3), a marker of autophagy, in livers taken from the patients with PBC $(n=37)$ and control livers $(n=75)$. We also examined the co-localization of LC3 with autophagy-related cathepsin D, lysosome-associated membrane protein-1 (LAMP-1), and senescent markers, $\mathrm{p} 16^{\mathrm{INK} 4 \mathrm{a}}$ and $\mathrm{p} 21^{\mathrm{WAF} 1 / \mathrm{Cip} 1}$. We examined the effect of autophagy inhibitor (3-methyladenine) on the induction of cellular senescence and senescence-associated secretion (CCL2 and CX3CL1) in cultured murine BECs. The expression of LC3 was specifically seen in vesicles in BECs in the inflamed and damaged small bile ducts in PBC, when compared with non-inflamed small bile ducts in PBC and in control livers $(P<0.01)$. The expression of LC3 was closely related to the expression of cathepsin D, LAMP-1, and senescent markers. In cultured BECs, oxidative stress, DNA damage, and serum deprivation induced cellular senescence, when compared with control and the inhibition of autophagy significantly decreased the stress-induced cellular senescence $(P<0.01)$. Furthermore, the secretion level of CCL2 and CX3CL1 increased significantly by various stress and suppressed by the inhibition of autophagy $(P<0.01)$. In conclusion, autophagy is specifically seen in the damaged small bile ducts along with cellular senescence in PBC. The inhibition of autophagy suppressed cellular senescence in cultured cells. These findings suggest that autophagy may mediate the process of biliary epithelial senescence and involve in the pathogenesis of bile duct lesions in PBC.

Laboratory Investigation (2010) 90, 835-843; doi:10.1038/labinvest.2010.56; published online 8 March 2010

KEYWORDS: autophagy; cellular senescence; p16 ${ }^{\text {INK4a }} ;$ p2 $1^{\text {WAF1/Cip1 }} ;$ primary biliary cirrhosis

Autophagy and cellular senescence are two distinct cellular responses to stress. An appropriate cellular stress response is critical for maintaining tissue integrity and function and for preventing diseases. Cells respond to stress with adaptation, repair, and recovery, or are diverted into irreversible cell-cycle exit (senescence) or are eliminated through programmed cell death (apoptosis). ${ }^{1}$ Autophagy is a genetically regulated program responsible for the turnover of cellular proteins and damaged organelles. This evolutionarily conserved process is characterized by the formation of double membrane cytosolic vesicles, autophagosomes, which sequester cytoplasmic content and deliver it to lysosomes. ${ }^{2,3}$ Autophagy is often associated with acute metabolic changes and rapid protein replacement. Microtubule-associated proteins-light chain $3 \beta$
(LC3), a homolog of autophagy-related protein 8, which is essential for autophagy and associated to the autophagosome membranes after processing, is a widely used marker of autophagy. ${ }^{4,5}$ Autophagy can enable adaptation to stress through the degradation of cellular proteins and organelles to suppress damage, maintain metabolism, and promote cellular viability and fitness. ${ }^{1,6}$

Cellular senescence is a state of stable cell arrest with active metabolism. Similar to apoptosis, senescence can be a failsafe program against a variety of cellular insults. In contrast to apoptosis, however, in which the cytotoxic signals converge to a common mechanism, senescence is typically a delayed stress response involving multiple effector mechanisms. These effector mechanisms include epigenetic regulation, ${ }^{7}$

Department of Human Pathology, Kanazawa University Graduate School of Medicine, Kanazawa, Japan

Correspondence: Dr Y Nakanuma, MD, Department of Human Pathology, Kanazawa University Graduate School of Medicine, Kanazawa 920-8640, Japan.

E-mail: pbcpsc@kenroku.kanazawa-u.ac.jp

Received 29 September 2009; revised 13 January 2010; accepted 14 January 2010 
the DNA damage response, ${ }^{8}$ and the senescence-associated secretory phenotypes (SASP). ${ }^{9-11}$ Although autophagy can delay apoptosis, a function for autophagy in senescence was not earlier known. ${ }^{1}$ Recent studies disclosed that autophagy is induced during and facilitates the process of senescence. ${ }^{4}$

Primary biliary cirrhosis (PBC) is an organ-specific autoimmune disease and presents with chronic, progressive cholestasis, and liver failure. ${ }^{12-14} \mathrm{PBC}$ is characterized histologically as a cholangitis of small bile ducts (chronic non-suppurative destructive cholangitis, CNSDC) eventually followed by the extensive loss of small bile ducts. ${ }^{13-15}$ Although there have been many studies on the immunopathological features, ${ }^{16-18}$ there have been few studies on the pathogenesis of bile duct loss in PBC. We have reported cellular senescence of biliary epithelial cells (BECs) with the augmented expression of senescence-associated $\beta$-galactosidase (SA$\beta$-gal), ${ }^{19} \mathrm{p} 16^{\mathrm{INK} 4 \mathrm{a}}$, and $\mathrm{p} 21^{\mathrm{WAF} 1 / \mathrm{Cip} 1}$, and telomere shortening in damaged small bile ducts in PBC. We have suggested that the cellular senescence may be involved in the pathogenesis of progressive bile duct loss in $\mathrm{PBC} .^{20-22}$ A possible association of oxidative stress and decreased expression of Bmil polycomb ring finger oncogene (Bmil) was suggested to be involved in the pathogenesis of cellular senescence in PBC. ${ }^{20-23}$

Although it is well known that autophagy has important functions in clearing misfolded proteins in hepatocytes in liver diseases such as $\alpha-1$ antitrypsin deficiency and hypofibrinogenemia, ${ }^{24}$ there are few studies regarding autophagy in BECs and its relation between liver diseases to our knowledge. Given that BECs in damaged small bile ducts in PBC show senescent features, we examined an involvement of autophagy in the process of biliary epithelial senescence in PBC. Furthermore, we examined whether the inhibition of autophagy influences the induction of senescence phenotypes in cultured mouse BECs. This study suggests the involvement of autophagy in the induction of cellular senescence in BECs and may contribute to the pathogenesis of PBC.

\section{MATERIALS AND METHODS Human Study}

\section{Classification of intrahepatic biliary tree}

The intrahepatic biliary tree is classified into the intrahepatic large and small bile ducts (septal and interlobular bile ducts) by their size and distributions in the portal tracts. ${ }^{25}$ In this study, septal and interlobular bile ducts are termed as small bile ducts. Bile ductules, which are characterized by tubular or glandular structures with poorly defined lumen and located at the periphery of the portal tracts, ${ }^{25,26}$ are not included in the small bile ducts.

Small bile ducts were histologically divided largely into 'inflamed' and non-inflamed. Inflamed bile ducts include the bile ducts showing biliary epithelial damages and being surrounded and occasionally infiltrated by inflammatory cells such as CNSDC in $\mathrm{PBC}^{14}$ and also the bile ducts embedded in infiltrating lymphoid cells and showing mild epithelial damages in chronic viral hepatitis $(\mathrm{CVH})$ (hepatitis duct lesion).

\section{Liver tissue preparation}

A total of 112 liver tissue specimens (all were biopsied or surgically resected) were collected from the liver disease file of our laboratory and affiliated hospitals. The Ethics Committee in Kanazawa University approved this study. The liver specimens enrolled in this study were $37 \mathrm{PBC}, 22 \mathrm{CVH}, 22$ non-alcoholic steatohepatiits, 10 livers with extrahepatic biliary obstruction (EBO), and 21 'histologically normal' livers. All $\mathrm{PBC}$ were from the patients fulfilling the clinical, serological, and histological characteristics consistent with the diagnosis of $\mathrm{PBC}{ }^{14} \mathrm{PBC}$ livers were staged histologically, ${ }^{27}$ and 26 and 10 of PBC were of stages 1,2 (early PBC) and of stages 3, 4 (advanced PBC), respectively. Eleven CVH were regarded as F0-2 and 11 as F3, 4, respectively. ${ }^{28}$ Two and 20 of CVH cases were serologically positive for hepatitis B surface $B$ antigen and anti-hepatitis $C$ viral antibody, respectively. Causes of EBO were obstruction of the bile duct at the hepatic hilum or the extrahepatic bile ducts, because of carcinoma or stone, and the duration of jaundice was $<1$ month. 'Histologically normal' livers were obtained from surgically resected livers for traumatic hepatic rupture or metastatic liver tumor. The liver tissues used were taken from the part sufficiently away from the trauma and tumor.

Liver tissue samples were fixed in $10 \%$ neutral-buffered formalin, and embedded in paraffin. More than 20 serial sections, $4 \mu \mathrm{m}$ thick, were cut from each block. Several were processed routinely for histopathologic study, and the remainder was processed for the following immunohistochemistry.

\section{Immunohistochemistry}

We examined immunohistochemically the expression of LC3, a widely used autophagy marker. In addition, we examined immunohistochemically the expression of autophagy-related lysosomal protein, cathepsin D, lysosome-associated membrane protein-1 (LAMP-1), and senescence-associated markers (p16 ${ }^{\mathrm{INK} 4 \mathrm{a}}$ and $\left.\mathrm{p} 21^{\mathrm{WAF1} / \mathrm{Cip} 1}\right)$. Immunostaining was performed using the antibodies shown in Table 1, as described earlier. ${ }^{21}$ In brief, after pretreatment for antigen retrieval as described in Table 1, blocking endogenous peroxidase, the sections were incubated with the primary antibody at $4^{\circ}$ overnight. The Envision + solution for mouse (Dako) or Histofine Simple Stain MAX-PO (G) (Nichirei, Tokyo, Japan) was then applied for $30 \mathrm{~min}$ at room temperature. The reaction products were visualized using 3-3'diaminobenizidine tetra-hydrochloride (Sigma Chemica, St Louis, MO, USA) and $\mathrm{H}_{2} \mathrm{O}_{2}$. The sections were then lightly counterstained with methyl green or hematoxylin. A similar dilution of the control mouse or goat immunoglobulin G (Dako) was applied instead of the primary antibody as negative control. Positive and negative controls were routinely 
Table 1 Primary antibodies used in this study

\begin{tabular}{|c|c|c|c|c|}
\hline Primary antibody & Type (clone) & Pretreatment & Dilution & Source \\
\hline LC3 & Goat poly & $\mathrm{MW}-\mathrm{CB}\left(95^{\circ} \mathrm{C}, 20 \mathrm{~min}\right)$ & $1: 50$ & Santa-Cruz, Santa-Cruz, CA, USA \\
\hline LAMP-1 & Mouse mono (E-5) & $\mathrm{MW}-\mathrm{CB}\left(95^{\circ} \mathrm{C}, 20 \mathrm{~min}\right)$ & $1: 200$ & Santa-Cruz, Santa-Cruz, CA, USA \\
\hline p16 $6^{\text {INK4a }}$ & Mouse mono (JC8) & eARI-BA $\left(121^{\circ} \mathrm{C}, 5 \mathrm{~min}\right)$ & $1: 100$ & Neomarkers, Freemont, CA, USA \\
\hline
\end{tabular}

LC3, microtubule-associated proteins-light chain 3 $\beta$; LAMP-1, lysosome-associated membrane protein-1; MW, microwave treatment; CB, $0.05 \mathrm{M}$ citric buffer ( $\mathrm{pH}$ 6); eARl, electronic antigen retrieval instrument (pascal, Dako); BA, $0.05 \mathrm{M}$ boric acid buffer ( $\mathrm{pH} 8$ ).

included. When more than three cells showed positive signal, in a small bile duct, the bile duct was regarded as positive.

\section{Double immunostaining}

We also performed double immunostaining for LC3 with senescence markers, $\mathrm{p} 16^{\mathrm{INK} 4 \mathrm{a}}$ and $\mathrm{p} 21^{\mathrm{WAF} 1 / \mathrm{Cip} 1}$. In brief, LC3 was detected as described above, followed by second staining for either of the senescence markers ( $\mathrm{p} 16^{\mathrm{INK} 4 \mathrm{a}}$ and p2 $1^{\text {WAF1/Cip1 } 1}$ ) using Vector Blue Alkaline Phosphatase Substrate kit (Vector Laboratories).

\section{Culture Study}

Cell culture and treatments of mouse intrahepatic BECs

Mouse intrahepatic BECs were isolated from 8-week-old female BALB/c mice and were purified and cultured as described earlier. ${ }^{29}$ The maintenance and passage of cultured BECs was made as described earlier. ${ }^{29}$ The cell density of the cells was $<80 \%$ during experiments. In several experiments, BECs were treated with hydrogen peroxide (PO, $100 \mu \mathrm{M}$ for $2 \mathrm{~h}$ ), washed throughout to remove $\mathrm{PO}$, and cultured in fresh medium. DNA damage was induced by a treatment with Etoposide (100 $\mu \mathrm{M}$, Sigma Chemica). In several experiments, 3-methyladenine (3MA, $5 \mathrm{mM}$, Sigma Chemica), an inhibitor of the class III phosphatidylinositol 3-kinase (class III PI3K) complex involved in initial autophagosome formation, was used to inhibit autophagy. ${ }^{30}$

\section{Immunofluorescence staining for cultured cells}

The BECs growing in a Lab-Tek chamber were fixed with 5\% buffered formalin and cold methanol for 20 min each. After blocking with 3\% BSA, the cells were incubated with either of the primary antibodies for LC3 (described above) overnight. The cells were then incubated with Alexa-546-labeled rabbit anti-goat IgG (Molecular probes, Eugene, OR, USA) for $30 \mathrm{~min}$, counterstained with $10 \mu \mathrm{g} / \mathrm{ml} 4^{\prime}$ and 6-diamidino-2phenylindole, and evaluated under a conventional fluorescence microscope (Olympus, Tokyo, Japan).

\section{Assay for cellular senescence}

SA- $\beta$-gal activity was detected by using the senescence detection kit (BioVision, Mountain View, CA, USA) according to manufacturer's protocol. ${ }^{19}$ The proportion of senescent cells in each condition was assessed at Day 4 by counting the percentage of SA- $\beta$-gal-positive cells in at least $1 \times 10^{3}$ total cells using light microscopy.

Enzyme-linked immunosorbent assay of CCL2 and CX3CL1 Recent studies suggest that senescent cells modulate microenvironment by expressing SASP, including inflammatory cytokines and chemokines, such as CXCL8/interleukin (IL)-8, CCL2/monocyte chemotactic protein-1 (MCP)-1, and etc. $^{9-11}$ We also found that CX3CL1/fractalkine is one of SASP (paper in submission). Therefore, we examined whether the inhibition of autophagy influence the induction of CCL2/MCP-1 and CX3CL1 as senescence phenotypes; SASP. After $1 \times 10^{4}$ BECs were seeded on 12 -well plates for $24 \mathrm{~h}$, they were treated with PO, Etoposide, or serum deprivation with or without 3MA for 4 days. The concentration of CCL2/ MCP-1 and that of CX3CL1 in culture media were measured by enzyme-linked immunosorbent assay (ELISA) using an Immunoassay kits ( $\mathrm{R}$ and $\mathrm{D}$ Systems), according to the manufacturer's instructions.

\section{Immunoblotting}

The cell lysate samples $(15 \mu \mathrm{l})$ were resolved by SDS-PAGE and transferred to a nitrocellulose membrane as described earlier. ${ }^{31}$ After transfer, the membranes were processed for immunoblotting as described earlier. ${ }^{31}$ The primary antibody for LC 3 was described above. The $\alpha$-tubulin was detected by using mouse monoclonal anti- $\alpha$-tubulin (clone TU-01, Zymed, South San Francisco, CA, USA).

\section{Statistical analysis}

Statistical analysis for the difference used the Wilcoxon rank sum test. When the $P$-value was $<0.05$, the difference was regarded as significant. 


\section{RESULTS}

\section{Human Study}

Increased expression of autophagy marker LC3 in the damaged BECs in PBC

The expression of LC3 was specifically seen in cytoplasmic vesicles in BECs in the inflamed and damaged small bile ducts in PBC, whereas the expression of LC3 was absent or faint in normal livers (Figure 1a). The expression of LC3 was significantly more frequent and intense in the inflamed small bile ducts in PBC, when compared with noninflamed small bile ducts in PBC and small bile ducts in control livers $(P<0.01)$ (Table 2$)$. The expression of cathepsin D and LAMP-1, autophagy-related lysosomal proteins, was also seen in cytoplasmic vesicles in the inflamed and damaged bile ducts in PBC (Figure 1b). Double immunostaining revealed that the expression of LC3 was closely co-localized with the expression of senescent markers $\mathrm{p} 16^{\mathrm{INK} 4 \mathrm{a}}$ and $\mathrm{p} 21^{\mathrm{WAF} 1 / \mathrm{Cip} 1}$ in BECs in the damaged bile ducts in PBC (Figure 2). The co-expression of LC3 and $\mathrm{p} 21^{\mathrm{WAF} 1 / \mathrm{Cip} 1}$ was more frequently observed when compared with the co-expression of LC3 and $\mathrm{p} 16^{\mathrm{INK} 4 \mathrm{a}}$ in the damaged bile ducts in PBC.

The vesicular expression of LC3 was observed in ductular reactions in $\mathrm{PBC}$, to various degrees, whereas it was rarely seen in ductular reactions in control livers. We have reported that a part of ductular cells in ductular reaction shows cellular senescence as well as the damaged small bile ducts in PBC. ${ }^{22}$ Taken together, it is plausible that the vesicular expression of LC3 in ductular reaction may be associated with higher rate of senescent ductular cells in PBC. Further studies are needed to clarify the relation between the cell kinetics of 'bipotential stem/progenitor cells' and autophagy and/or cellular senescence in ductular reaction. The vesicular expression of LC3 was not observed in large bile ducts in PBC and control livers examined.

\section{Culture Study}

Induction of autophagy by several factors inducing cellular senescence

We determined whether autophagy is induced by several factors that are known to induce cellular senescence in BECs. ${ }^{20,21}$ LC3 is a widely used marker of autophagy and the lipid-conjugated form of LC3 (LC3-II) localized to the membrane of autophagosomes. It can be separated from the non-conjugated form (LC3-I) by immunoblotting. Immunoblotting showed that LC3-II was upregulated by the treatment with PO $(100 \mu \mathrm{M})$, Etoposide $(100 \mu \mathrm{M})$, and serum deprivation (Figure 3a). Immunofluorescence showed that vesicular cytoplasmic expression of LC3 was upregulated by serum deprivation, whereas the expression of LC3 was faint in control (no treatment) (Figure 3b). The upregulated expression of LC3 by serum deprivation was inhibited by a treatment with the autophagy inhibitor, 3MA (5 mM) (Figure 3b).

\begin{tabular}{|c|c|c|c|c|}
\hline Diseases & $\begin{array}{l}\text { Number } \\
\text { of patients }\end{array}$ & Type of BD & $\begin{array}{l}\text { Number } \\
\text { of } B D\end{array}$ & $\begin{array}{c}\text { Number of BD } \\
\text { with vesicular } \\
\text { LC3 expression (\%) }\end{array}$ \\
\hline \multirow[t]{2}{*}{ PBC } & 37 & Inflamed & 126 & $114(90.5 \%)^{*}$ \\
\hline & & Non-inflamed & 109 & $30(27.5 \%)^{\star *}$ \\
\hline \multirow[t]{2}{*}{$\mathrm{CVH}$} & 22 & Inflamed & 98 & $6(6.1 \%)^{* *}$ \\
\hline & & Non-inflamed & 134 & 0 \\
\hline \multirow[t]{2}{*}{ NASH } & 22 & Inflamed & 15 & $2(13.3 \%)^{* *}$ \\
\hline & & Non-inflamed & 147 & $1(0.7 \%)$ \\
\hline \multirow[t]{2}{*}{ EBO } & 10 & Inflamed & 15 & $1(6.7 \%)^{* *}$ \\
\hline & & Non-inflamed & 79 & 0 \\
\hline Normal liver & 21 & Non-inflamed & 188 & 0 \\
\hline
\end{tabular}

LC3, microtubule-associated proteins-light chain $3 \beta$; PBC, primary biliary cirrhosis; $\mathrm{CVH}$, chronic viral hepatitis; NOSH, non-alcoholic steatohepatitis; EBO, extrahepatic biliary obstruction; $\mathrm{BD}$, bile duct.

${ }^{\star} P<0.01$ versus $\mathrm{PBC}$, non-inflamed and other groups; ${ }^{*} P<0.01$ versus normal livers.

\section{Inhibition of autophagy significantly decreased cellular senescence}

Percentage of cells positive for SA- $\beta$-gal was significantly higher in cultured BECs treated with $\mathrm{H}_{2} \mathrm{O}_{2}$ (SA- $\beta$-gal-labeling index, 37.0 \pm 13.1 ), Etoposide (34.0 \pm 5.7$)$, and serum deprivation $(34.2 \pm 11.7)$, when compared with control without treatment $(3.9 \pm 1.4)(P<0.01)$ (Figure $4 \mathrm{a})$. Percentage of cells positive for SA- $\beta$-gal was significantly decreased by a treatment with the autophagy inhibitor 3MA $(5 \mathrm{mM}) \quad(\mathrm{PO}+3 \mathrm{MA}, \quad 5.1 \pm 4.5 ; \quad$ Etop $+3 \mathrm{MA}, \quad 8.6 \pm 2.9$; Dep + 3MA, 6.9 \pm 4.8$)(P<0.01) \quad($ Figure $4 \mathrm{a})$. These data suggest that autophagy may be involved in the process of cellular senescence induced by oxidative stress, DNA damage, and serum deprivation.

\section{Inhibition of autophagy significantly decreased SASPS}

ELISA showed that the secretion of CCL2, one of SASPs, was increased in BECs treated with PO $(2.74 \pm 0.1 \mathrm{pg} / \mathrm{ml})$, Etoposide $(2.01 \pm 0.25 \mathrm{pg} / \mathrm{ml})$, and serum deprivation $(35.17 \pm 1.98 \mathrm{pg} / \mathrm{ml})$, when compared with control $(1.3 \pm 0.1 \mathrm{pg} / \mathrm{ml}) \quad(P<0.01) \quad$ (Figure 4b). The enhanced secretion of CCL2 was significantly decreased by a treatment with the autophagy inhibitor $3 \mathrm{MA}(5 \mathrm{mM})(\mathrm{PO}+3 \mathrm{MA}$, $0.0 \pm 0.0 ;$ Etop + 3MA, 0.0 $\pm 0.0 ;$ Dep + 3MA, 6.64 \pm 1.32$)$ $(P<0.01)$ (Figure $4 \mathrm{~b})$. The secretion of CX3CL1 was also increased in BECs treated with serum deprivation $(553.9 \pm 43.8 \mathrm{pg} / \mathrm{ml})$, when compared with control $(96.1 \pm 16.5 \mathrm{pg} / \mathrm{ml}) \quad(P<0.01)$. The enhanced secretion of CX3CL1 was significantly decreased by a treatment with the autophagy inhibitor 3MA (5 mM) (Dep + 3MA, 258.3 \pm 9.20; Cont $+3 \mathrm{MA}, 0.1 \pm 0.1) \quad(P<0.01) \quad$ (Figure $4 \mathrm{~b})$. The autophagy inhibitor $3 \mathrm{MA}$ indeed acts through inhibition of 
a

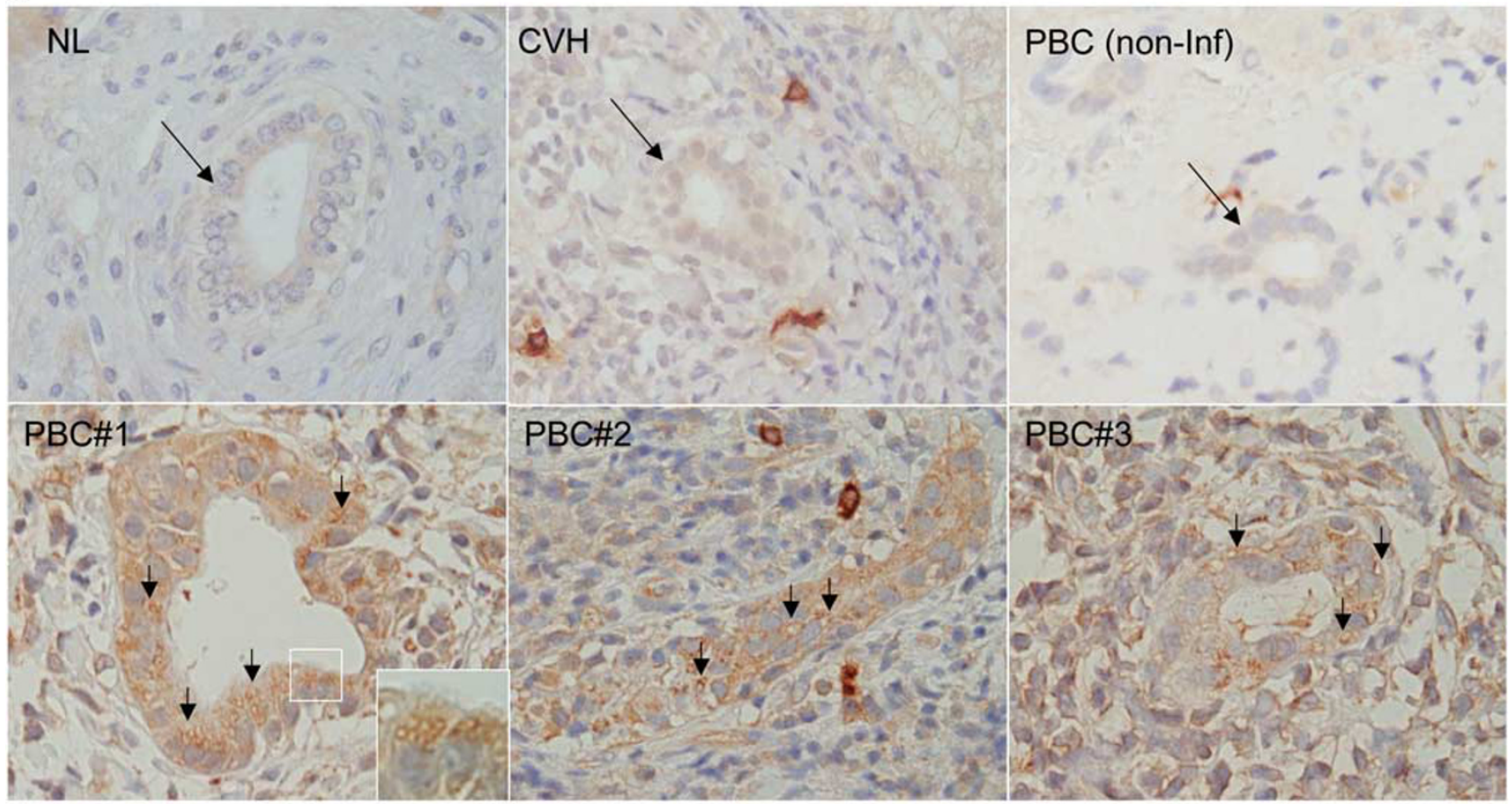

b
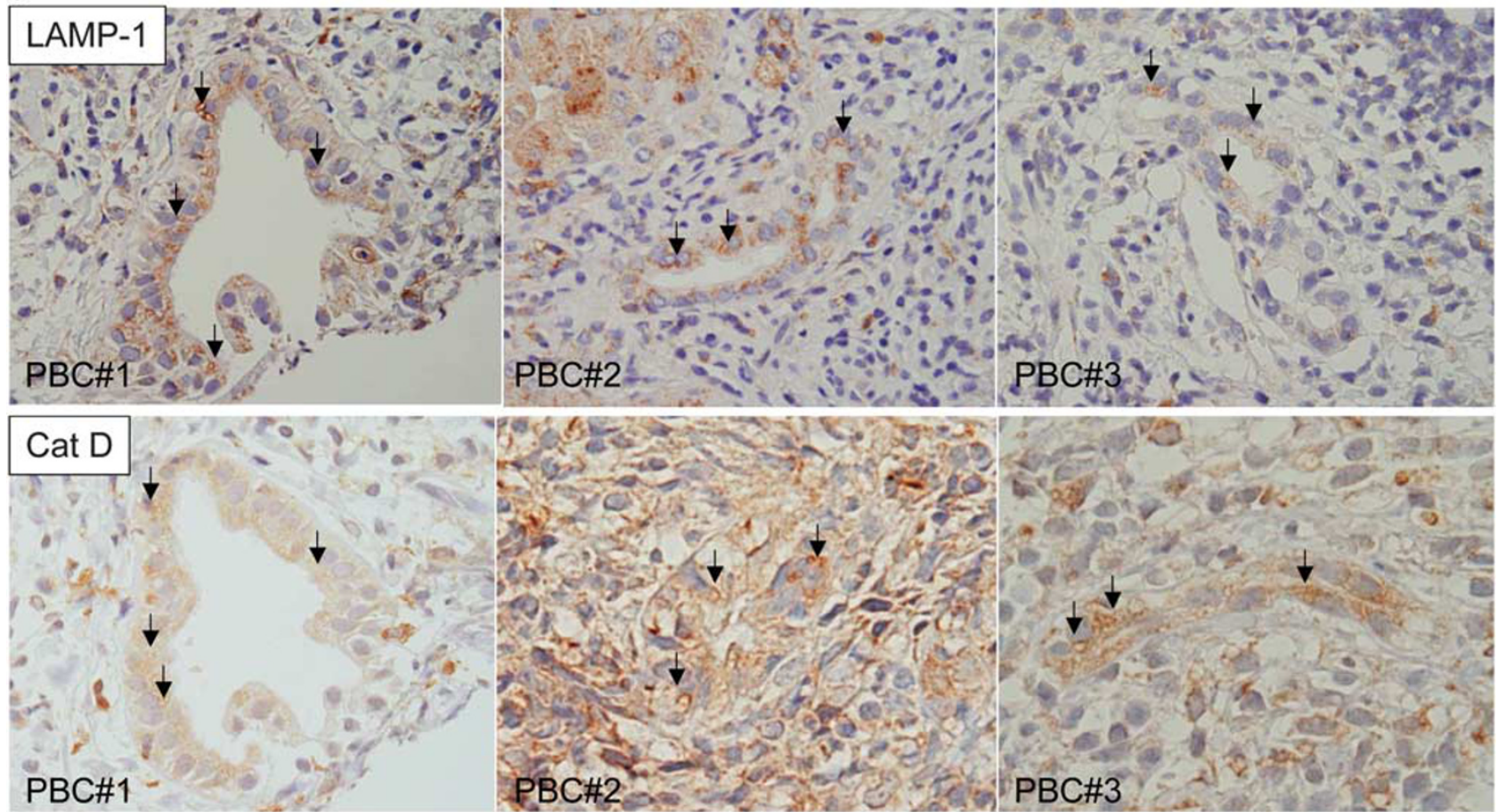

Figure 1 Increased vesicular expression of autophagy marker LC3 in the damaged small bile ducts in PBC. (a) The expression of LC3 was absent in BECs in small bile duct (arrow) in normal liver (NL, top left), CVH (top middle) and non-inflamed bile duct in PBC (top right). The expression of LC3 was detected in intracytoplasmic vesicles (short arrows) around nuclei in the damaged small bile duct in PBC (bottom). PBC cases \#1-3 (stage 2). Immunostaining for LC3. Original magnification, $\times 400$ (Inset, $\times 1000)$. (b) Top: the expression of lysosome-associated membrane protein (LAMP)-1 was detected in intracytoplasmic vesicles (short arrows) in the damaged small bile duct (arrows) in PBC. PBC cases \#1-3 (stage 2). Immunostaining for LAMP-1. Original magnification, $\times 400$. Bottom: the expression of autophagy-related lysosomal protein: cathepsin $D(C a t D)$ was detected in intracytoplasmic vesicles (short arrows) in the damaged small bile duct (arrows) in PBC. PBC cases \#1-3 (stage 2). Immunostaining for cathepsin D. Original magnification, $\times 400$. 

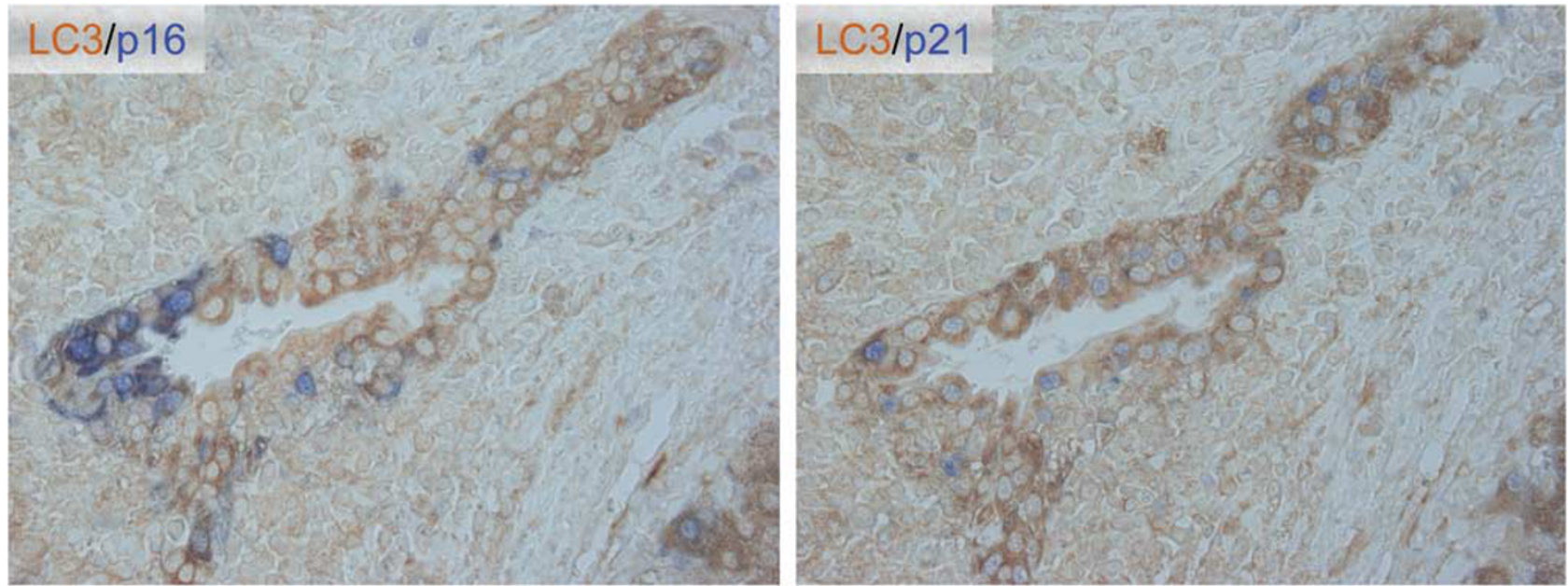

Figure 2 Co-localization of LC3 with senescent markers $\mathrm{p} 16^{\mathrm{INK} 4 a}$ and $\mathrm{p} 21^{\text {WAF1/Cip } 1}$ in the damaged small bile ducts in PBC. Nuclear and cytoplasmic expression of senescent marker p16 $16^{\mathrm{INK} 4 \mathrm{a}}$ was seen in a part of BECs showing cytoplasmic vesicular expression of LC3 in the damaged bile ducts in PBC (left). Nuclear expression of senescent marker $\mathrm{p} 21^{\mathrm{WAF} 1 / \mathrm{Cip} 1}$ was also seen in a part of BECs in the same damaged bile ducts in PBC (right). PBC, stage 2. Double

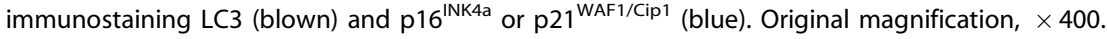

Figure 3 Increased expression of autophagy marker LC3 in cultured BECs with oxidative stress, DNA damage, and serum deprivation. (a) Oxidative stress and DNA damage were induced by a $2 \mathrm{~h}$ treatment with PO $(100 \mu \mathrm{M})$ and a $48 \mathrm{~h}$ treatment with Etoposide (Etop, $100 \mu \mathrm{M}$ ). Autophagosomeassociating form of LC3 (LC3-II) was upregulated by oxidative stress, DNA damage, and serum deprivation (Dep). LC3-I, a cytosolic form of LC3; $\alpha$-tub, $\alpha$-tubulin; Cont, control. (b) Immunofluorescence for the autophagy marker LC3 (left column) and a counter stain with 6-diamidino-2-phenylindole (DAPI) (right column). Vesicular cytoplasmic expression of LC3 was upregulated by serum deprivation (Dep, $48 \mathrm{~h}$ ), whereas the expression of LC3 was faint in control (no treatment). The upregulated expression of LC3 by serum deprivation was inhibited by a treatment with $3 \mathrm{MA}(5 \mathrm{mM})$.

cellular senescence rather than be a direct general effect on chemokine release, as the treatment with 3MA did not affect CXCL1 chemokine release induced by lipopolysaccharide (Supplementary Information).

\section{DISCUSSION}

The data obtained in this study are summarized as follows: (1) The vesicular cytoplasmic expression of LC3, an autophage marker, was characteristically seen in the inflamed damaged small bile ducts in PBC. Cathepsin D and LAMP-1, the autophagy-related lysosomal proteins, was also co-expressed in the inflamed and damaged small bile ducts, suggesting that autophagy is involved in the pathogenesis of PBC. (2) The expression of LC3 was co-localized with the expression of senescent markers $\mathrm{p} 16^{\mathrm{INK} 4 \mathrm{a}}$ and $\mathrm{p} 21^{\mathrm{WAF} 1 / \mathrm{Cip} 1}$ in the inflamed damaged small bile ducts in PBC. (3) Oxidative stress, DNA damage, and serum deprivation induced autophagy and cellular senescence in cultured BECs. An autophagy inhibitor, 3MA, inhibited significantly the stressinduced cellular senescence in cultured BECs. (4) Oxidative stress, DNA damage, and serum deprivation upregulated a
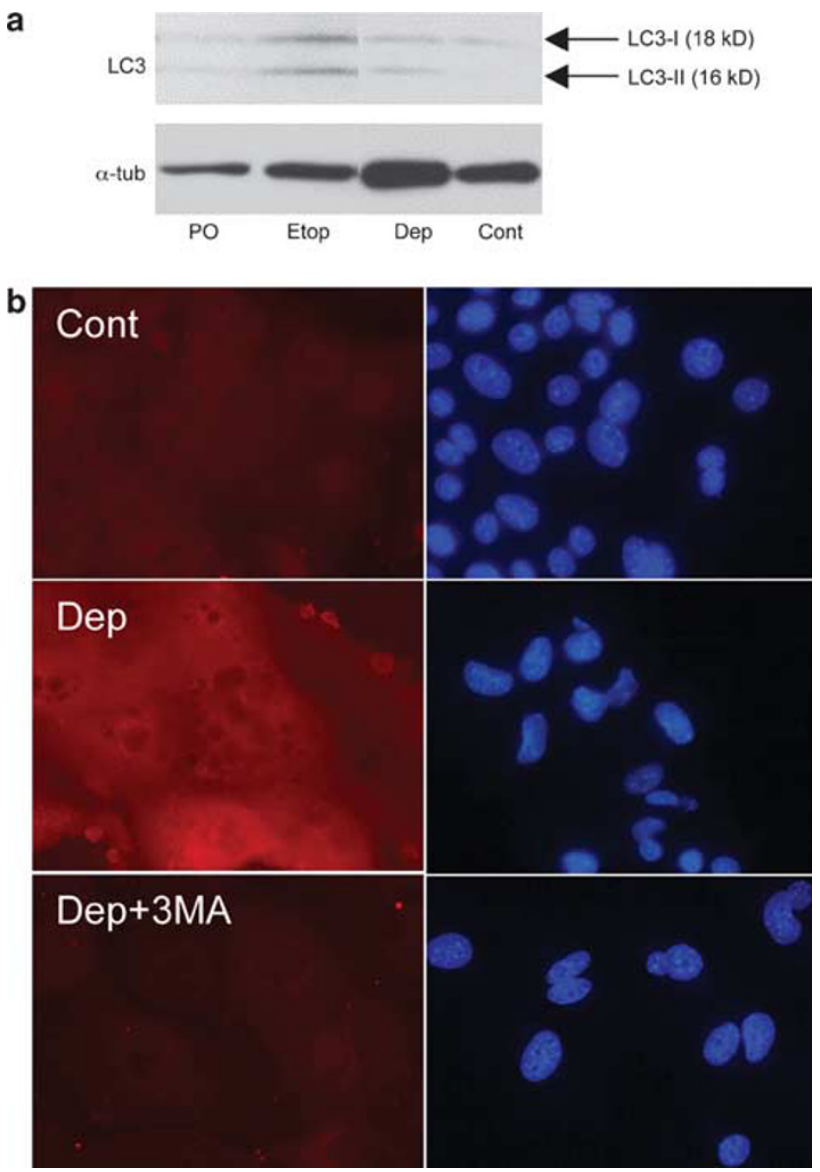

SASP, CCL2, and CX3CL1 in BECs, and a treatment with 3MA decreased the SASP in BECs treated with various stress.

This study first disclosed that autophagy may be involved in the bile duct lesion in PBC. A representative autophagy 
a
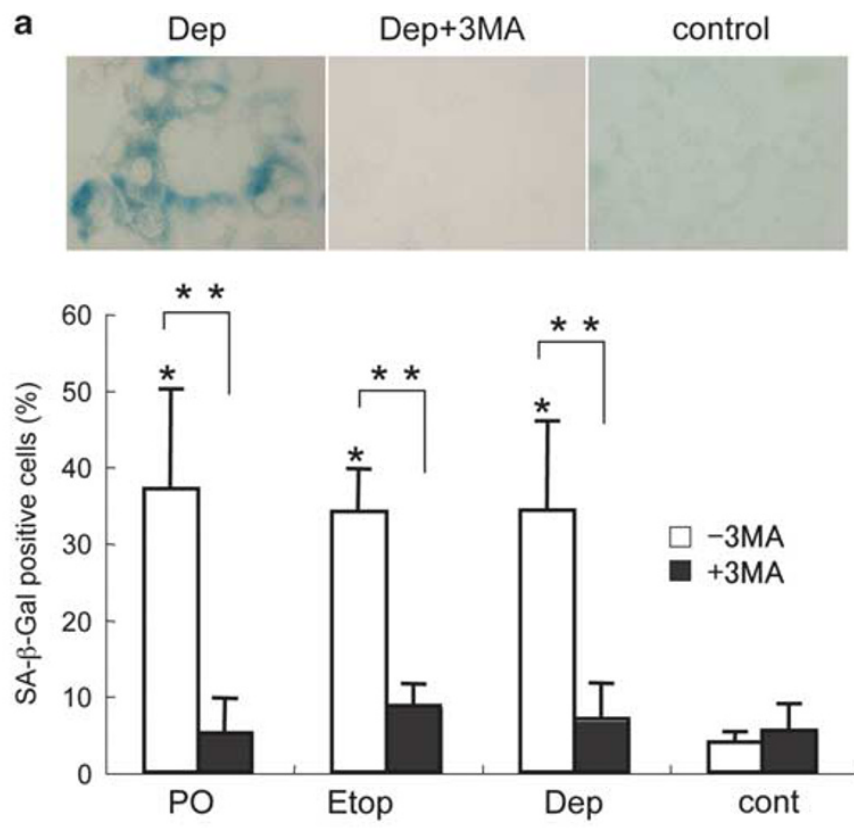

CCL2

b

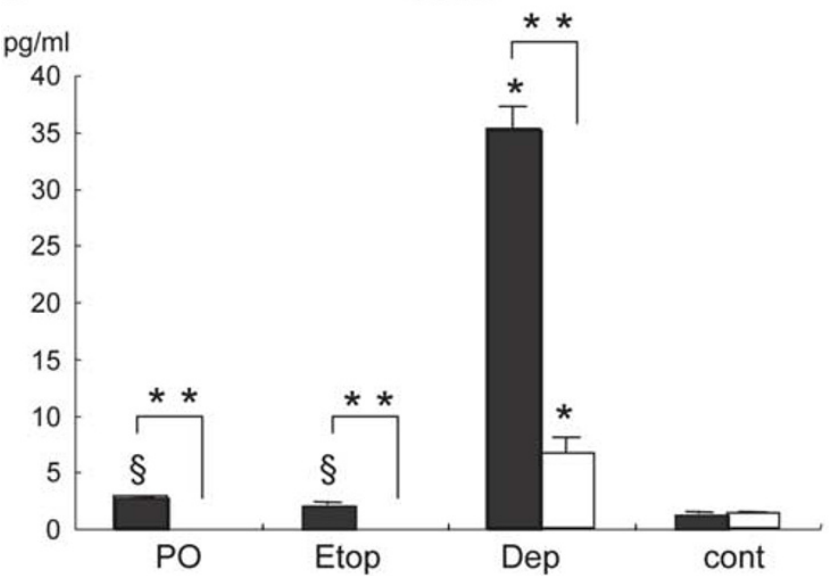

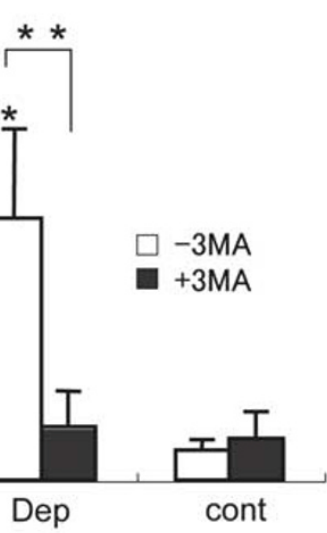

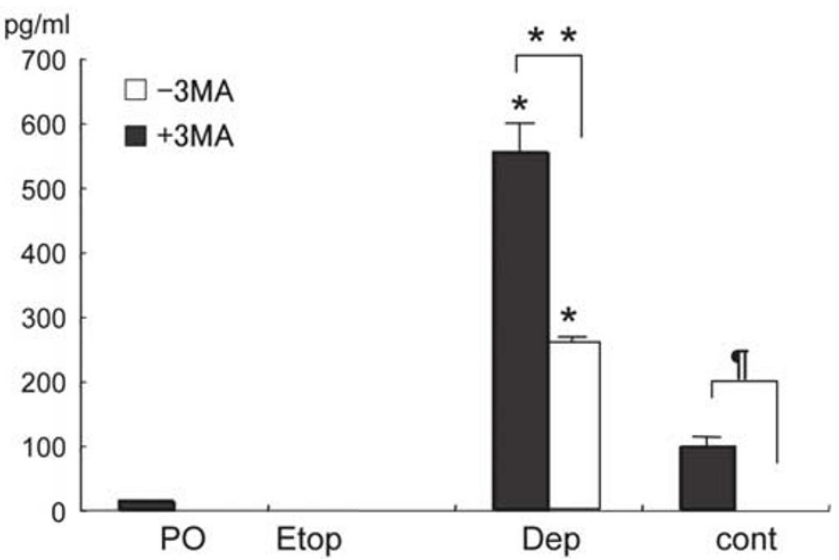

Figure 4 Inhibition of autophagy significantly decreased cellular senescence. (a) Cellular senescence was assessed by SA- $\beta$-gal on Day 4 after a 2 -h treatment with PO $(100 \mu \mathrm{M})$, a treatment with Etoposide (Etop, $100 \mu \mathrm{M})$ and serum deprivation (Dep). Percentage of cells positive for SA- $\beta$-gal was significantly higher in cells treated with PO (SA- $\beta$-gal-labeling index, $37.0 \pm 13.1$ ), Etoposide ( $34.0 \pm 5.7)$, and serum deprivation (34.2 \pm 11.7$)$, when compared with control without treatment (3.9 \pm 1.4$)$. Percentage of cells positive for $S A-\beta$-gal was significantly decreased by a treatment with the autophagy inhibitor $3 \mathrm{MA}(5 \mathrm{mM})(\mathrm{PO}+3 \mathrm{MA}, 5.1 \pm 4.5$; Etop $+3 \mathrm{MA}, 8.6 \pm 2.9$; Dep $+3 \mathrm{MA}, 6.9 \pm 4.8)$. Data was expressed as mean \pm s.d. ${ }^{\star} P<0.01$ compared with control, ${ }^{*} P<0.01$. (b) Left: the secretion of CCL2, an SASP was increased in BECs treated with PO $(2.74 \pm 0.1 \mathrm{pg} / \mathrm{ml})$, Etoposide $(2.01 \pm 0.25 \mathrm{pg} / \mathrm{ml})$, and serum deprivation $(35.17 \pm 1.98 \mathrm{pg} / \mathrm{ml})$, when compared with control $(1.3 \pm 0.1 \mathrm{pg} / \mathrm{ml})$. The enhanced secretion of CCL2 was significantly decreased by a treatment with the autophagy inhibitor 3MA (5 mM) (PO + 3MA, $0.0 \pm 0.0$; Etop + 3MA, $0.0 \pm 0.0$; Dep + 3MA, $6.64 \pm 1.32$ ). Right: the secretion of CX3CL1 was increased in BECs treated with serum deprivation $(553.9 \pm 43.8 \mathrm{pg} / \mathrm{ml})$, when compared with control $(96.1 \pm 16.5 \mathrm{pg} / \mathrm{ml})$. The enhanced secretion of CX3CL1 was significantly decreased by a treatment with the autophagy inhibitor $3 \mathrm{MA}(5 \mathrm{mM})(\mathrm{Dep}+3 \mathrm{MA}, 258.3 \pm 9.20$; Cont $+3 \mathrm{MA}, 0.1 \pm 0.1)$. ${ }^{\star} P<0.01$ and ${ }^{\S} P<0.05$ compared with control, ${ }^{*} p<0.01, " P<0.05$.

marker LC3 was characteristically expressed in cytoplasmic vesicles in the damaged small bile ducts in PBC. Although the LC3 antibody we used in this study reacts with both a cytoplasmic and a membrane-associated form of LC3 (LC3-I and LC3-II, respectively), it is plausible that the vesicular LC3 expression exactly corresponds to a membraneassociated form of LC3 (LC3-II), which localize to the membrane of autophagosomes. The expression of LC3 was significantly higher in inflamed small bile ducts in PBC, when compared with non-inflamed bile ducts and other control livers. The expression of cathepsin D, an autophagyrelated lysosomal enzyme, was coordinately expressed in the damaged bile ducts with LC3. There is no study reporting the presence and involvement of autophagy in BECs in biliary diseases, so far. In hepatocytes, it is well known that autophagy has important functions in the balance of 
energy and nutrients for basic cell functions and the removal of misfolded proteins in liver diseases such as $\alpha-1$ antitrypsin deficiency and hypofibrinogenemia. ${ }^{24}$ This study indicates that autophagy is induced in BECs in diseased condition in $\mathrm{PBC}$ and may be related to the pathogenesis of the disease.

It is of interest that an earlier report showed phagocytosis of apoptotic BECs in PBC. ${ }^{32}$ In this report, the authors showed the increased BEC phagocytosis of apoptotic BECs in $\mathrm{PBC}$ and other liver diseases and also in cultured rat BECs. ${ }^{32}$ They raised a possibility that phagocytosed apoptotic cells may consequently be an exogenous source of autoantigens in BECs. The phagocytosed apoptotic cells in BECs in this earlier report appear to be completely different from the increased autophagy in BECs in PBC disclosed in this study. The phagocytosed apoptotic cells were observed in healthy BECs in PBC and the size of the phagocytosed apoptotic cells was about one quarter of nucleus in BECs, ${ }^{32}$ whereas the autophagic vesicles were observed in BECs in the damaged bile ducts in $\mathrm{PBC}$ in this study and their size was far smaller than 'the phagocytosed apoptotic cells.' It is possible that the autophagic vesicles may contain a special form of mitochondrial autoantigens as well as the apoptotic cells ${ }^{32}$ and that it may influence the autoimmune reactions in PBC. Further studies are necessary to clarify the significance of increased autophagy in BECs in the autoimmune conditions in PBC.

Furthermore, this study disclosed that autophagy in BECs is closely co-localized with cellular senescence in the inflamed and damaged bile ducts in PBC. We have reported cellular senescence of BECs with the augmented expression of SA- $\beta$ gal, ${ }^{19} \mathrm{p} 16^{\mathrm{INK} 4 \mathrm{a}}$, and $\mathrm{p} 21^{\mathrm{WAF1} / \mathrm{Cip} 1}$, and telomere shortening in damaged small bile ducts in PBC. We have suggested that the cellular senescence may be involved in the pathogenesis of progressive bile duct loss in PBC. ${ }^{20-22}$ Taken together, it is plausible that autophagy and cellular senescence may coordinately have a function in the pathogenesis in PBC. The co-expression of LC3 and p $21^{\text {WAF1/Cip1 }}$ was more frequently observed, when compared with the co-expression of LC3 and p $16^{\mathrm{INK} 4 \mathrm{a}}$ in the damaged bile ducts in PBC in this study. $\mathrm{p} 16^{\mathrm{INK} 4 \mathrm{a}}$ and $\mathrm{p} 21^{\mathrm{WAF} 1 / \mathrm{Cip} 1}$ work coordinately in process of the cellular senescence and $\mathrm{p} 21^{\mathrm{WAF} 1 / \mathrm{Cip} 1}$ is involved in the induction of senescence, whereas $\mathrm{p} 16^{\mathrm{INK} 4 \mathrm{a}}$ is involved in maintenance of senescence, reportedly. ${ }^{33}$ Therefore, closer co-expression of the autophagic marker LC 3 and $\mathrm{p} 21^{\mathrm{WAF} 1} /$ ${ }^{\mathrm{Cip} 1}$ suggest that the process of cellular senescence may follow the process of autophagy.

This study disclosed for the first time that autophagy may be involved in the process of cellular senescence in cultured BECs. Interestingly, autophagy inhibitor 3MA suppressed the induction of cellular senescence and the induction of SASP in BECs with oxidative stress, DNA damage, and serum deprivation. Recently, Young et $a l^{4}$ identified autophagy as a new effector mechanism of senescence. Autophagy is activated during senescence and its activation is correlated with negative feedback in the mammalian target of rapamycin pathway. A subset of autophagy-related genes is upregulated during senescence: over-expression of one of those genes, ULK3, induced autophagy and senescence. ${ }^{4}$ Furthermore, inhibition of autophagy delays the senescence phenotype, including senescence-associated secretion. ${ }^{4}$ This study supported the earlier study by Young et al ${ }^{4}$ and provides evidence that autophagy may mediate cellular senescence in the inflamed and damaged small bile ducts in PBC.

Recent data suggest that senescent cells have an important function in modulating microenvironment by expressing SASP such as cytokines (IL-6, IL-1, and etc), chemokines (IL-8, CCL2/MCP-1, and etc), growth factors, and profibrogenic factors. ${ }^{9-11}$ In fact, studies in human with biliary disorders and in animal models of biliary fibrosis have shown that BECs can express a number of profibrogenic and chemotactic proteins such as IL-8 and CCL2/MCP-1, the latter capable of attracting and activating cells of both inflammatory and stellate cell lineage. ${ }^{34}$ Upregulation of several cytokines and chemokines in the damaged bile ducts in PBC has been reported, so far, ${ }^{35-37}$ and recent studies disclosed that most of these factors are known to belong to SASP. ${ }^{9-11}$ This study clearly showed that SASP such as CCL2/MCP-1 and CX3CL1 is induced by various stress such as oxidative stress and serum deprivation in cultured BECs through autophagy followed by cellular senescence. CCL2/ MCP-1 and CX3CL1 are potent chemotactic factors inducing accumulation of inflammatory cells such as monocytes and T cells. Therefore, it is plausible that SASP secreted by senescent BECs may modulate microenvironment around bile duct and may accentuate inflammation and fibrosis in PBC.

In conclusion, autophagy is specifically seen in the senescent BECs in PBC. The inhibition of autophagy suppressed cellular senescence and senescence-associated secretion of CCL2/MCP-1 and CX3CL1 in cultured BECs. These findings suggest that autophagy may mediate biliary epithelial senescence and involved in the pathogenesis of bile duct lesions in PBC.

Supplementary Information accompanies the paper on the Laboratory Investigation website (http://www.laboratoryinvestigation.org)

\section{ACKNOWLEDGEMENT}

This study was supported in part by a Grant-in Aid for Scientific Research (C) from the Ministry of Education, Culture, Sports and Science and Technology of Japan (18590325 and 2590366).

\section{DISCLOSURE/CONFLICT OF INTEREST}

The authors declare no conflict of interest.

1. White E, Lowe SW. Eating to exit: autophagy-enabled senescence revealed. Genes Dev 2009;23:784-787.

2. Mizushima N, Levine B, Cuervo AM, et al. Autophagy fights disease through cellular self-digestion. Nature 2008;451:1069-1075.

3. Ohsumi Y. Molecular dissection of autophagy: two ubiquitin-like systems. Nat Rev Mol Cell Biol 2001;2:211-216.

4. Young AR, Narita $M$, Ferreira $M$, et al. Autophagy mediates the mitotic senescence transition. Genes Dev 2009;23:798-803. 
5. Kabeya Y, Mizushima N, Ueno T, et al. LC3, a mammalian homologue of yeast Apg8p, is localized in autophagosome membranes after processing. EMBO J 2000;19:5720-5728.

6. Levine B, Kroemer G. Autophagy in the pathogenesis of disease. Cell 2008;132:27-42.

7. Narita $M$, Krizhanovsky $V$, Nunez $S$, et al. A novel role for high-mobility group a proteins in cellular senescence and heterochromatin formation. Cell 2006;126:503-514.

8. Bartkova J, Rezaei N, Liontos M, et al. Oncogene-induced senescence is part of the tumorigenesis barrier imposed by DNA damage checkpoints. Nature 2006;444:633-637.

9. Acosta JC, O'Loghlen A, Banito $A$, et al. Chemokine signaling via the CXCR2 receptor reinforces senescence. Cell 2008;133: 1006-1018.

10. Kuilman T, Michaloglou C, Vredeveld LC, et al. Oncogene-induced senescence relayed by an interleukin-dependent inflammatory network. Cell 2008;133:1019-1031.

11. Wajapeyee N, Serra RW, Zhu X, et al. Oncogenic BRAF induces senescence and apoptosis through pathways mediated by the secreted protein IGFBP7. Cell 2008;132:363-374.

12. Gershwin ME, Mackay IR, Sturgess $A$, et al. Identification and specificity of a CDNA encoding the $70 \mathrm{kd}$ mitochondrial antigen recognized in primary biliary cirrhosis. J Immunol 1987;138:3525-3531.

13. Kaplan M. Primary biliary cirrhosis. New Engl J Med 1996;335: 1570-1580.

14. Portmann $B$, Nakanuma $Y$. Diseases of the bile ducts. In: MacSween $R$ Burt A, Portmann B, Ishak K, Scheuer P, Anthony P (eds). Pathology of the Liver, 4th Eds edn. Churchill Livingstone: London, 2001, pp 435-506.

15. Nakanuma Y, Ohta G. Histometric and serial section observations of the intrahepatic bile ducts in primary biliary cirrhosis. Gastroenterology 1979;76:1326-1332.

16. Fussey $\mathrm{S}$, Guest J, James $\mathrm{O}$, et al. Identification and analysis of the major M2 autoantigens in primary biliary cirrhosis. Proc Natl Acad Sc USA 1988;85:8654-8658.

17. Kita $H$, Matsumura $\mathrm{S}, \mathrm{He} X \mathrm{~S}$, et al. Quantitative and functional analysis of PDC-E2-specific autoreactive cytotoxic T lymphocytes in primary biliary cirrhosis. J Clin Invest 2002;109:1231-1240.

18. Shimoda S, Van de Water J, Ansari A, et al. Identification and precursor frequency analysis of a common $T$ cell epitope motif in mitochondrial autoantigens in primary biliary cirrhosis. J Clin Invest 1998:102:1831-1840.

19. Dimri GP, Lee $X$, Basile $G$, et al. A biomarker that identifies senescent human cells in culture and in aging skin in vivo. Proc Natl Acad Sci USA 1995;92:9363-9367.

20. Sasaki M, lkeda $H_{\text {, Haga }} \mathrm{H}$, et al. Frequent cellular senescence in small bile ducts in primary biliary cirrhosis: a possible role in bile duct loss. J Pathol 2005:205:451-459.

21. Sasaki M, Ikeda H, Sato $Y$, et al. Decreased expression of Bmi1 is closely associated with cellular senescence in small bile ducts in primary biliary cirrhosis. Am J Pathol 2006;169:831-845.
22. Sasaki $\mathrm{M}$, Ikeda $\mathrm{H}$, Yamaguchi J, et al. Telomere shortening in the damaged small bile ducts in primary biliary cirrhosis reflects ongoing cellular senescence. Hepatology 2008;48:186-195.

23. Sasaki M, Ikeda $H$, Nakanuma Y. Activation of ATM signaling pathway is involved in oxidative stress-induced expression of mito-inhibitory p21(WAF1/Cip1) in chronic non-suppurative destructive cholangitis in primary biliary cirrhosis: an immunohistochemical study. J Autoimmun 2008;31:73-78

24. Yin XM, Ding WX, Gao W. Autophagy in the liver. Hepatology 2008;47:1773-1785.

25. Nakanuma Y, Sasaki M. Expression of blood-group-related antigens in the intrahepatic biliary tree and hepatocytes in normal livers and various hepatobiliary diseases. Hepatology 1989;10:174-178.

26. Roskams TA, Theise ND, Balabaud C, et al. Nomenclature of the finer branches of the biliary tree: canals, ductules, and ductular reactions in human livers. Hepatology 2004;39:1739-1745.

27. Ludwig J. Small-duct primary sclerosing cholangitis. Semin Liver Dis 1991;11:11-17.

28. Desmet V, Gerber M, Hoofnagle J, et al. Classification of chronic hepatitis: diagnosis, grading and staging. Hepatology 1994;19:1513-1520.

29. Katayanagi K, Kono N, Nakanuma Y. Isolation, culture and characterization of biliary epithelial cells from different anatomical levels of the intrahepatic and extrahepatic biliary tree from a mouse. Liver 1998;18:90-98.

30. Seglen PO, Gordon PB. 3-Methyladenine: specific inhibitor of autophagic/lysosomal protein degradation in isolated rat hepatocytes. Proc Natl Acad Sci USA 1982;79:1889-1892.

31. Sasaki M, Van De Water J, Kenny TP, et al. Immunoglobulin gene usage and immunohistochemical characteristics of human monoclonal antibodies to the mitochondrial autoantigens of primary biliary cirrhosis induced in the XenoMouse. Hepatology 2001;34(4 Part 1):631-637.

32. Allina J, Hu B, Sullivan DM, et al. T cell targeting and phagocytosis of apoptotic biliary epithelial cells in primary biliary cirrhosis. J Autoimmun 2006;27:232-241.

33. Stein GH, Drullinger LF, Soulard A, et al. Differential roles for cyclindependent kinase inhibitors p21 and p16 in the mechanisms of senescence and differentiation in human fibroblasts. Mol Cell Biol 1999;19:2109-2117.

34. Alvaro D, Mancino MG, Glaser $S$, et al. Proliferating cholangiocytes: a neuroendocrine compartment in the diseased liver. Gastroenterology 2007;132:415-431.

35. Yasoshima M, Kono N, Sugawara $\mathrm{H}$, et al. Increased expression of interleukin-6 and tumor necrosis factor-alpha in pathologic biliary epithelial cells: in situ and culture study. Lab Invest 1998;78:89-100.

36. Tsuneyama K, Harada K, Yasoshima M, et al. Monocyte chemotactic protein-1, -2 , and -3 are distinctively expressed in portal tracts and granulomata in primary biliary cirrhosis: implications for pathogenesis. J Pathol 2001:193:102-109.

37. Shimoda S, Harada K, Niiro H, et al. Biliary epithelial cells and primary biliary cirrhosis: the role of liver-infiltrating mononuclear cells. Hepatology 2008;47:958-965. 